\title{
PAGOD syndrome
}

INSERM

\section{Source}

INSERM. (1999). Orphanet: an online rare disease and orphan drug data base. $\underline{P A G O D}$ syndrome. ORPHA:991

PAGOD syndrome is a severe developmental syndrome characterized by multiple congenital anomalies including cardiovascular defects, pulmonary hypoplasia, diaphragmatic defects and genital anomalies. 